A. Pramesh Rao, G. Swarup and Gopal-Krishna, eds.

\title{
Cosmic Microwave Background at Low Frequencies
}

\author{
R. Subrahmanyan \\ Australia Telescope National Facility, CSIRO, Locked bag 194, Narrabri, \\ NSW 2390, Australia
}

\begin{abstract}
The next generation low-frequency radio telescopes may probe cosmological models by means of observations of the cosmic microwave background (CMB). I discuss the prospects for observations of CMB imprints - recombination lines from the epoch of recombination, $\mu$ distortions and angular temperature anisotropies - at low frequencies. A future low-frequency radio telescope, like the proposed SKA, may be capable of attempting some difficult CMB measurements because of the large collecting area and large element numbers; however, this will require a telescope design that will allow specialized calibration strategies and will give emphasis to the control of spurious responses.
\end{abstract}

\section{Introduction}

The coming decade may well see a monumental enhancement in observing capabilities at low radio frequencies if, for example, the proposed square-kilometre array (SKA) is constructed. The quantum leap in the total collecting area and in the numbers of antenna elements constituting the array could move certain key observational proposals from the realm of 'dreams' to reality. In this context - and while the telescope configuration and antenna element design are still being debated - it is perhaps timely that key and challenging experiments that may be of relevance to the emerging opportunity are highlighted so that the instrument specifications may be tailored to eventually allow their endeavor.

The discovery of the existence of the cosmic microwave background (CMB), followed by refinements in measurements of its characteristics leading to the precise $C O B E$ observations of its spectrum and anisotropies, have been a major constraint and discriminant between models of cosmology and structure formation. Almost all measurements that have been valuable in constraining cosmology theories have been made to-date at $\mathrm{cm}, \mathrm{mm}$ and sub-mm wavelengths. The long wavelength measurements of the CMB have been plagued by large errors owing to the bright Galactic background temperatures and high levels of extragalactic foreground discrete source confusion.

In this article, I shall attempt to highlight some aspects of CMB research and discuss their relevance to low-frequency radio astronomy in the context of the changed scenario expected in the coming decade if the SKA is constructed. Possible observing strategies and calibration schemes are introduced; implications for the specifications for the SKA are touched upon. 


\section{The CMB temperature at low frequencies}

The COBE-FIRAS experiment (Fixen et al. 1996) measured the temperature of the cosmic microwave background (CMB) in the frequency range 70-640 GHz: no significant distortions from a Plankian form were detected and the best-fit thermodynamic temperature of the cosmic background was determined to be $2.728 \pm 0.004 \mathrm{~K}$.

Within the framework of the hot big bang cosmology, structure in the universe is believed to have formed via gravitational instabilities from primordial 'seed' density perturbations. The damping of sub-horizon scale pressure waves as perturbations in the radiation field enter the horizon over the redshift interval about $5 \times 10^{6}$ to $5 \times 10^{5}$ - are expected to inevitably leave their imprint as a $\mu$ distortion in the CMB (Daly 1991; Hu, Scott, \& Silk 1994). The wavelength $\lambda_{\max }$ at which maximum distortion occurs is approximately $\lambda_{\text {max }} \simeq 2.2\left(\Omega_{b} h^{2}\right)^{-2 / 3} \mathrm{~cm}$ (Burigana, Danese, \& De Zotti 1991); for $\Omega_{b} h^{2} \simeq 0.019$ (Burles, Nottel \& Turner 1999), we may expect the temperature distortion to be a maximum at $30 \mathrm{~cm}$ wavelength. The value of the chemical potential $\mu_{\circ}-$ as a consequence of the damping of primordial density perturbations - may be as small as $10^{-8}$ if the index of the COBE-DMR normalized matter power spectrum has an index $n=1$, but could be as large as $10^{-4}$ if $n \simeq 1.6$ (Hu, Scott \& Silk 1994): correspondingly, the maximum temperature distortion may be as large as $0.01 \mathrm{~K}$. Separately, any release of radiant energy in the redshift interval $5 \times 10^{6}>z>5 \times 10^{5}$, perhaps owing to the decay of particles with half lives in this range of cosmic times, would also result in $\mu$ distortions (Silk \& Stebbins 1983).

The COBE-FIRAS measurements of the CMB spectrum limit $|\mu|$ to be less than $9 \times 10^{-5}$ (Fixen et al. 1996). This implies that we may not expect a deviation exceeding about $0.008 \mathrm{~K}$ at metre wavelengths as a consequence of any $\mu$ distortion. Interestingly, these constraints placed on $\mu$ by the extremely precise $C O B E-F I R A S$ measurements are at least an order of magnitude more valuable than the results from long-wavelength measurements of the CMB spectrum, although they may have been made at frequencies where the deviation may be a maximum.

Recent measurements of the CMB temperature at frequencies close to $1 \mathrm{GHz}$ include the $600 \mathrm{MHz}$ estimate of $T_{C M B}=3.0 \pm 1.2 \mathrm{~K}$ by Sironi et al. (1990), the $1400 \mathrm{MHz}$ estimate of $T_{C M B}=2.65 \pm 0.3 \mathrm{~K}$ by Staggs et al. (1996) and the $1470 \mathrm{MHz}$ estimate of $T_{C M B}=2.26 \pm 0.19 \mathrm{~K}$ by Bensadoun et al. (1993). A more recent estimate, albeit with larger errors, is that $T_{C M B}=3.45 \pm 0.78 \mathrm{~K}$ at $1280 \mathrm{MHz}$ (Raghunathan \& Subrahmanyan 2000).

The large uncertainties in long-wavelength measurements of the absolute brightness of the CMB are due, in part, to the relatively high brightness of the Galactic background at these wavelengths, the larger size of the receiving elements, the greater difficulty in cooling them to cryogenic temperatures and consequently the greater contribution from losses in the antenna and associated feed. The ground-based measurements also suffer from uncertainty associated with estimations of the atmospheric contribution. Improvements in estimates of $T_{C M B}$ at low frequencies may come from improved methods of reducing losses and/or developing methods of cancelling unwanted contributions, making multi- 
frequency measurements over an extremely wide frequency range, and by placing the apparatus above the atmosphere.

In this context it may be mentioned that the experimental setup of Raghunathan \& Subrahmanyan used a novel technique of selecting cable lengths to cancel, via destructive interference, an unwanted contribution from the cold load connected to the third port of the circulator. The DIMES project (see the website at ceylon.gsfc.nasa.gov/DIMES), with a wide spectral coverage from 2 to $100 \mathrm{GHz}$ and based on a satellite platform, may be the kind of experiment that could improve sensitivity to $\mu$ distortions by more than an order of magnitude.

\section{Fine structure in the CMB spectrum}

The cooling of the primeval plasma in the expanding universe is expected to have led to recombination at a temperature near $3000 \mathrm{~K}$ (Peebles 1968; Jones \& Wyse 1985). It has been argued (Bartlett \& Stebbins 1991) that measurements to-date of the CMB spectrum (that limit free-free emission from an ionized intergalactic medium and $y$ distortions arising from Compton scattering by hot electrons in such a medium) do not require a neutral period and hence do not rule out the possibility that the universe remained ionized throughout its history. However, the upper limits on the redshift of re-ionization (post recombination) derived from the position of the peak in the spectrum of CMB anisotropies (Griffiths, Barbosa, \& Liddle 1999) indicates that the universe was perhaps largely neutral beyond a redshift of about 40: the data supports primordial recombination. In this context, a direct observational probe of the recombination epoch would be valuable: the recombination lines that are inevitably generated during recombination is a potential probe.

The dominant additive recombination-line feature in the CMB spectrum is the $\mathrm{L}_{\alpha}$ hydrogen line, this is expected to manifest in a spectral 'hump' at about $0.014 \mathrm{~cm}$ wavelength and a broad 'continuum' extending to higher frequencies (Burdyuzha \& Chekmezov 1994).

Of interest to low-frequency radio astronomy are the hydrogen (and helium) recombination lines corresponding to transitions between highly excited states that may be visible today at metre and centimetre wavelengths owing to the extraordinary redshift $(z \sim 1100)$ of the epoch of recombination. As pointed out by Dubrovich \& Solyarov (1995) - continuing on the earlier work by Dubrovich (1975) and Bernshtein, Bernshtein, \& Dubrovich (1977) - the number of photons in transitions between adjacent levels as well as the ratio of the distance between consecutive lines to the line widths both decrease with increasing wavelength. As a consequence, the detection becomes extremely challenging at low frequencies. However, at least at $\mathrm{cm}$ wavelengths, the extragalactic background light is dominated by the CMB whose intensity varies inversely as the square of the wavelength; therefore, the ratio of line strength to background continuum may be expected to have a maximum at wavelengths $20-60 \mathrm{~cm}$. At these low frequencies, the line is expected (Dell'Antonio \& Rybicki 1993; Dubrovich \& Solyarov 1995) to appear as spectral features with peak-to-peak brightness of about $0.1 \mu \mathrm{K}$. The lines would be extremely broad because recombination is not 'instantaneous': the redshift interval $\Delta z / z$ over which the ionization fraction changes is about 20 per cent and is significantly greater than that obtained 
assuming quasi-equilibrium (Saha) ionization because recombination is 'stalled' and 'regulated' by the increased temperature in the Ly- $\alpha$ line as a consequence of recombination itself. This results in the spectral lines manifesting as spectral ripples with period 20 per cent of the observing frequency.

A detection of these spectral lines in the CMB would, apart from clarifying the thermal history of the baryons, place constraints on the baryon density and the mean matter density. These spectral features are of extremely low intensity; however, if radiant energy release in the early universe resulted in deviations in the radiation background from the Planckian form, the spectral features may have an increased prominence (Lyubarsky \& Sunyaev 1983). In this case their observation may provide information on early energy release.

\subsection{The observation of wideband CMB spectral features}

The detection of recombination lines or any other spectral features that were added to the CMB in the early universe is an extremely difficult and challenging experiment of the 'high-risk high-gain' type. It may be noted here that the spectral variations in temperature are expected to be smaller than the angular anisotropy in the CMB.

Clearly, the measurements will have to be made with a 'total-power' type telescope; any interferometer would resolve the uniform sky signal. Conventionally, 'total-power' sky spectra are obtained from auto-correlations of the single-dish signals measured over a range of delays. However, the receiver-noise component may be eliminated from the spectra by the use of 'correlation receivers': this will require a built-in capability for splitting the signal from the feeds before the front-end low-noise amplifiers. Alternately, 'total-power' sky spectra may be measured using an interferometer array by scalar averaging the cross-power spectral amplitudes.

Sensitivity The detection requires extremely high brightness sensitivity: the spectral features may be only $0.1 \mu \mathrm{K}$ and a factor $10^{-8}-10^{-9}$ of the system temperature. It is obvious that, despite the large bandwidths involved, the signals cannot be detected in any reasonable time using a single element telescope. However, because the signals are isotropic to a high degree, the antenna size is not directly a determinant of the sensitivity. Therefore, we may detect the signal by averaging the sky spectra from a large number of receivers behind relativelysmall spatially-separated antenna elements. If we assume that integration times of order $100 \mathrm{hr}$ are realistic (very much larger times would make the debugging of the system difficult), and that signals are to be detected with spectral resolution of order $50 \mathrm{MHz}$ at $1 \mathrm{GHz}$, the number of independent receivers is of order $10^{3}$ for a 3- $\sigma$ detection. Perhaps the elements of an SKA may be designed to attempt this experiment.

Wide bandwidth A second cause for difficulty with this experiment is the wide bandwidth required for the detection of these lines; receivers with low noise temperatures over a wide bandwidth exceeding 20 percent of the observing frequency are required. The receiver characteristics would undoubtedly change over this band and will have to be calibrated; however, spurious broadband spectral features may appear in the received spectrum as a result of any changes in the sky reception pattern over the band. Wide bandwidth observations, particularly at 
low frequencies, are also susceptible to radio interference: the experiment will require receivers with high dynamic range and interference excising/cancelling techniques.

Calibration Perhaps the greatest challenge would be the accurate calibration of the instrument response. Standard calibration techniques like beam switching fail because the signals are isotropic. Frequency switching may not be useful because no spectral region is line-free. Ideally what one would like to do to calibrate the instrument is to make separate observations with only the interesting signal 'switched off'.

A possible calibration strategy is to make the CMB spectral measurements using the individual elements of an array of telescopes, in 'total power' mode, and 'switch off' the uniform sky signal, for the purpose of calibration, by operating the array as an interferometer. The individual antenna spectral responses are determined from the spectral visibilities obtained while observing a strong, largely unresolved source, in interferometric mode. Such an approach may also allow calibration measurements to be made simultaneously with the total-power CMB spectral measurements: time variations in the band-pass characteristics would not then be a limiting factor.

In Fig. 1, I show an example where this calibration strategy has been applied to spectra of Galactic HI. These spectra were obtained with the Australia Telescope Compact Array (ATCA). Fig. 1(a) shows auto-correlation spectra obtained with the individual antennae, Fig. 1(b) shows the scalar-averaged crosspower spectra obtained by taking the spectral visibilities (these have been vector averaged on-line over $10 \mathrm{~s}$; within the narrow spectral bandwidth this averaging time is not enough to detect the sources in the field with signal-to-noise exceeding unity) on the interferometer baselines and averaging the amplitude spectra off-line. Fig. 1(c) shows the calibration spectrum obtained from the spectral visibilities measured on an unresolved calibrator. Fig. 1(d) and (e) show, respectively, the calibrated auto-correlation and scalar-averaged cross-power spectra. The signal-to-noise in the scalar-averaged spectra are worse because the scalar averaging of amplitudes was done only off line: the on-line $10 \mathrm{~s}$ averaging was performed vectorially in the individual $2-\mathrm{kHz}$ channels and, as a consequence, the amplitudes reduced by a factor 200 .

A disadvantage of such a scheme is that the calibration does not include the receiver noise characteristics; however, if the total-power spectra are obtained using correlation receivers, as discussed above, the spectra will not include a contribution from receiver noise. Another negative aspect of such an approach is that the calibration will require a strong source: the antenna temperature due to the calibrator source will have to be comparable to the system temperature if the calibration time should be comparable to the observing time.

Alternate methods of calibrating spectra, that may be worth exploring, are (i) using the 'Moon' to block the sky spectrum (Shaver et al. 1999; Stankevich, Wielebinski, \& Wilson 1970) and (ii) equalizing the instrument bandpass response by observing each sky spectral component through every spectrometer channel. The former may impose severe requirements on the linearity of the receivers and the latter method will require 'fine-tuning' capability in the first LO in the receiver chain. 

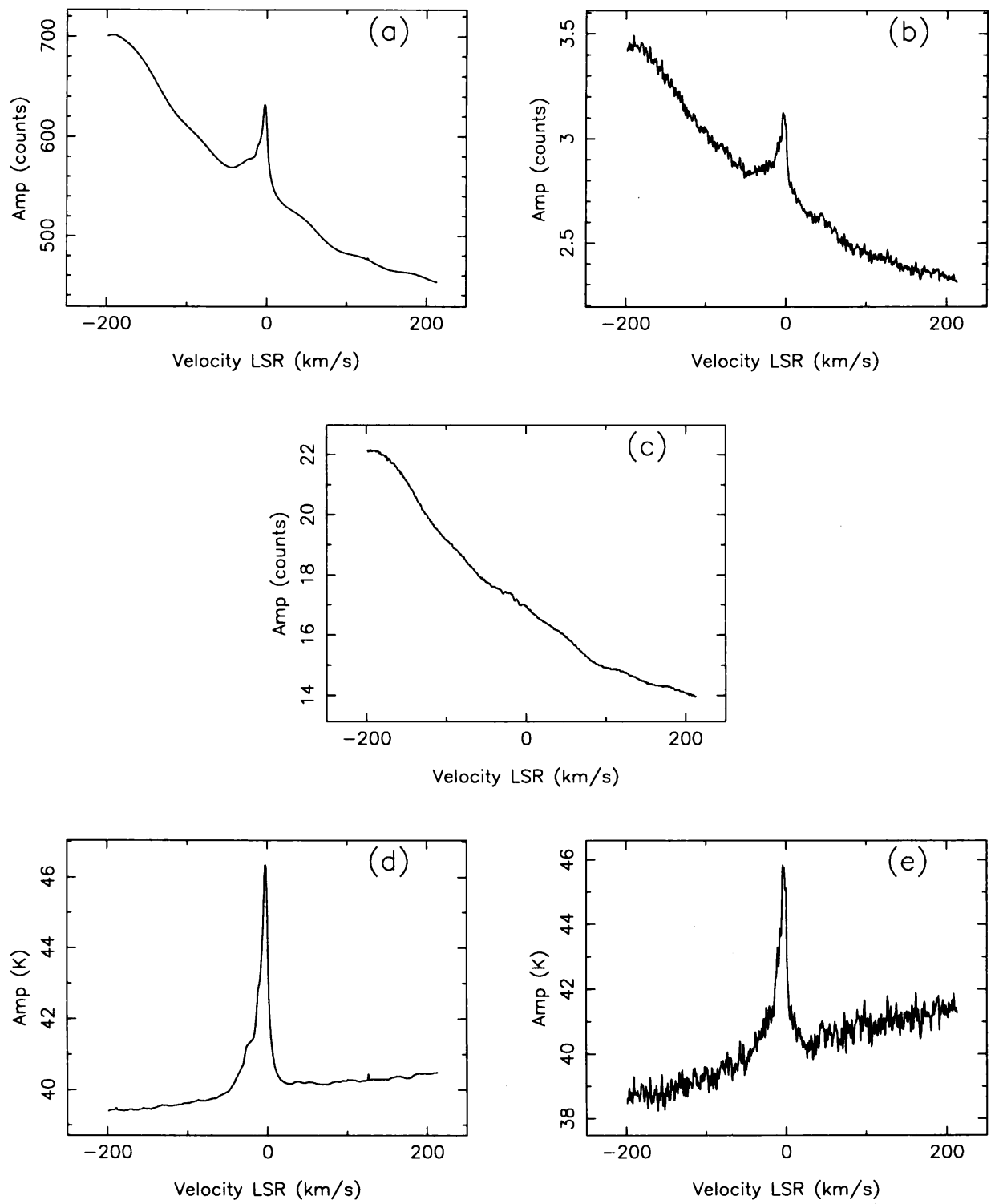

Figure 1. ATCA spectra of Galactic HI. (a) and (b) are, respectively, a single-dish auto-correlation spectrum and a scalar (amplitude) averaged cross-correlation spectrum. (c) is the bandpass calibration determined from interferometric observations of an unresolved calibrator. (d) and (e) are the corresponding calibrated spectra. 


\section{Angular anisotropies in the CMB temperature}

Most measurements of small-angle CMB anisotropy are being done at high radio frequencies (in the $15-90 \mathrm{GHz}$ range) where Galactic and extragalactic contaminants are a minimum. However, the high-frequency detections of small-angle anisotropy, which have to-date been Sunyaev-Zeldovich (S-Z) decrements towards clusters of galaxies, have been difficult measurements made with long integrations and using telescopes with small fields-of-view. All-sky images of CMB anisotropies with 10's of arcmin resolution are expected to become available in the coming decade from the $M A P$ and $P L A N C K$ satellite missions; however, they are expected to detect $\mathrm{S}-\mathrm{Z}$ anisotropies from only the relatively high-mass and nearby $\mathrm{S}-\mathrm{Z}$ clusters. The next generation of high-brightness-sensitive imaging arrays, like the CBI and AMIBA, which are specifically designed for surveys for S-Z clusters, are also expected to cover only small sky areas because of their small fields of view.

Owing to the upturn in the source counts at low flux density levels, the $\mu \mathrm{Jy}$ source counts at $\mathrm{GHz}$ frequencies are approximately (see, for example, Fig. 3 in Windhorst et al. 1993)

$$
n(S)=10^{8} S_{\mu \mathrm{Jy}}^{-2.2} f_{G H z}^{-0.8} \operatorname{arcmin}^{-2} \mathrm{Jy}^{-1} .
$$

In images made with a beam of FWHM $\theta$ arcmin, these sources may be expected to result in a 1- $\sigma$ confusion rms given by

$$
\Delta S=40 \theta^{1.7} f_{G H z}^{-0.7} \mu \mathrm{Jy}, \text { or } \Delta T=14 \theta^{0.3} f_{G H z}^{-2.7} \mathrm{mK} .
$$

The arcmin-resolution anisotropy searches made at frequencies $<10 \mathrm{GHz}$ including those with the VLA and the ATCA - have been limited by foreground discrete-source confusion. In this context, it is useful to ask whether a future low-frequency telescope, like the SKA, which will have the capability of imaging wide fields of view, could make large-area surveys for S-Z clusters and image the decrements with sufficient angular resolution to detect any sub-structure. The answer will depend on whether the enormous improvement in flux sensitivity in an SKA-type telescope will enable surveys for low-surface-brightness S-Z clusters below the confusion limit by detecting and subtracting a large part of the discrete-source confusion.

If we assume that all discrete sources above a lower flux density limit of $S_{m} \mu \mathrm{Jy}$ are subtracted from the sky images, the residual confusion $\mathrm{rms}$ is

$$
\Delta S=8 \theta f_{G H z}^{-0.4} S_{m}^{0.4} \mu \mathrm{Jy}, \text { or } \Delta T=3 \theta^{-1} f_{G H z}^{-2.4} S_{m}^{0.4} \mathrm{mK} .
$$

The proposed SKA is to have a sensitivity: $A_{\text {eff }} / T_{\text {sys }}=2 \times 10^{4} \mathrm{~m}^{2} \mathrm{~K}^{-1}$. We may expect the continuum images to have a thermal noise of about $50 \mathrm{nJy}$ with $10 \mathrm{hr}$ integration time. Assuming that foreground sources above $S_{m} \sim 250 \mathrm{nJy}$ are successfully subtracted, the residual confusion in arcmin resolution images may be expected to be as large as $2 \mathrm{mK}$ at $1 \mathrm{GHz}$ but as low as $6 \mu \mathrm{K}$ at $10 \mathrm{GHz}$.

Clearly, any useful survey for S-Z clusters at low frequencies, even with an $\mathrm{SKA}$, will require very long integration times and observations at frequencies $\gtrsim 10 \mathrm{GHz}$. However, because confusion will be an important limiting factor, it is important that particular attention be given to the design of the antenna 
element and array configuration in order to ensure that the sidelobe levels are low and roll off rapidly.

Acknowledgments. The Australia telescope is funded by the Commonwealth of Australia for operation as a National facility managed by CSIRO. The HI spectra displayed were obtained as part of collborative work with Mark Walker of the UNSW, Australia.

\section{References}

Bartlett, J.G. \& Stebbins, A. 1991, ApJ, 371, 8

Bensadoun, M., Bersanelli, M., De Amici, G., Kogut, A., Levin, S., Limon, M., Smoot, G.F., \& Witebsky, C. 1993, ApJ, 409, 1

Bernshtein, I.N., Bernshtein, D.N., \& Dubrovich, V.K. 1997, Sov. Astron., 21, 409

Burdyuzha, V.V. \& Chekmezov, A.N. 1994, Astron. rep., 38, 297

Burigana C., Danese, L., \& De Zotti, G. 1991, A\&A, 246, 49

Burles S., Nottel, K.M., \& Turner, M.S. 1999, astro-ph/9903300

Daly R. 1994, ApJ, 371, 14

Dell'Antonio, I.P. \& Rybicki, G.B. 1993, in Observational Cosmology, ed. G. Chincarini, A. Iovino, T. Maccacaro \& D. Maccagni, ASP Conf. Ser. Vol. 51,548

Dubrovich, V.K. 1975, Sov. Astron. Lett., 1, 196

Dubrovich, V.K. \& Stolyarov, V.A. 1995, A\&A, 302, 635

Fixen, D.J., Cheng, E.S., Gales, J.M., Mather, J.C., Shafer, R.A., \& Wright, E.L. 1996, ApJ, 486, 623

Griffiths, L.M., Barbosa, D., \& Liddle, A.R. 1999, MNRAS, 308, 854

Hu, W., Scott, D., \& Silk, J. 1994, ApJ, 430, L5

Jones, B.J.T. \& Wyse, R.F.G. 1985, A\&A, 149, 144

Lyubarsky, Y.E. \& Sunyaev, R.A. 1983, A\&A, 123, 171

Peebles, P.J.E. 1968, ApJ, 153, 1

Raghunathan, A. \& Subrahmanyan R. 2000, in preparation

Shaver, P.A., Windhorst, R.A., Madau, P., \& de Bruyn, A.G. 1999, A\&A, 345, 380

Silk, J. \& Stebbins, A. 1983, ApJ, 269, 1

Sironi, G., Limon, M., Marcellino, G., Bonelli, G., Bersanelli, M., Conti, G., \& Reif, K. 1990, ApJ, 357, 301

Staggs, S.T., Jarosik, N.C., Wilkinson, D.T., \& Wollack, E.J. 1996, ApJ, 458, 407

Stankevich, K.S., Wielebinski, \& R., Wilson, W.E. 1970, Aust. J. Phys., 23, 529

Windhorst R.A., Fomalont E.B., Partridge R.B., \& Lowenthal, J.D. 1993, ApJ, 405,498 